\title{
Chemische Analyse durch Spectralbeobachtungen
}

\author{
von
}

\author{
G. Kirchhoff und R. Bunsen
}

Annalen der Physik und Chemie, Bd. 110 No. 6, 1860, S. 161-189

Neu herausgegegeben von Gabriele Dörflinger,

Universitätsbibliothek Heidelberg, 2008.

http://www.ub.uni-heidelberg.de/archiv/15657

Der Text wurde durch ein Texterkennungsprogramm wiedergewonnen; die Seitenzählung des Originals ist am Seitenrand angegeben. 
Es ist bekannt, dass manche Substanzen die Eigenschaft haben, wenn sie in eine Flamme gebracht werden, in dem Spectrum derselben gewisse helle Linien hervortreten zu lassen. Man kann auf diese Linien eine Methode der qualitativen Analyse gründen, welche das Gebiet der chemischen Reactionen erheblich erweitert und zur Lösung bisher unzugänglicher Probleme führt. Wir beschränken uns hier zunächst nur darauf, diese Methode für die Metalle der Alkalien und alkalischen Erden zu entwickeln und ihren Werth an einer Reihe von Beispielen zu erläutern.

Die erwähnten Linien zeigen sich um so deutlicher, je höher die Temperatur und je geringer die eigene Leuchtkraft der Flamme ist. Die von Einem von uns angegebene Gaslampe ${ }^{1}$ liefert eine Flamme von sehr hoher Temperatur und sehr kleiner Leuchtkraft; dieselbe ist daher vorzugsweise geeignet $\mathrm{zu}$ Versuchen über die, jenen Substanzen eigentümlichen hellen Linien.

Auf Taf. V [s. Abb. 2] sind die Spectren dargestellt, welche die genannte Flamme giebt, wenn die so rein wie möglich dargestellten Chlorverbindungen von Kalium, Natrium, Lithium, Strontium, Calcium, Baryum in ihr verflüchtigt werden. Das Sonnenspectrum ist, um die Orientirung zu erleichtern, beigefügt.

Die zu den Versuchen benutzte Kaliumverbindung wurde durch Glühen von chlorsaurem Kali, welches zuvor sechs bis achtmal umkrystallisirt war, dargestellt.

Das Chlornatrium setzten wir aus reinem kohlensauren Natron und Salzsäure (162) zusammen, und reinigten dasselbe gleichfalls durch öfters wiederholtes Umkrystallisiren.

Das Lithionsalz war durch vierzehnmalige Fällung mit kohlensaurem Ammoniak gereinigt.

Zur Darstellung der Calciumverbindung diente ein möglichst reiner, in Salzsäure gelöster Marmor. Aus der Lösung desselben wurde durch fractionirte Fällung mit kohlensaurem Ammoniak kohlensaurer Kalk in zwei Portionen niedergeschlagen, von welchen nur die zuletzt niederfallende in salpetersauren Kalk verwandelt wurde. Das so erhaltene Kalksalz lösten wir zu wiederholten Malen in absolutem Alkohol auf und verwandelten es endlich nach Verflüchtigung des Alkohols und Fällung mit kohlensaurem Ammoniak durch Salzsäure in die Chlorverbindung.

Um das Chlorbaryuin rein zu erhalten, extrahirten wir die käufliche Verbindung zu wiederholten Malen durch Zusammenreiben und Kochen mit nicht ganz absolutem Alkohol. Der so extrahirte, von Alkohol befreite, in Wasser gelöste Rückstand ward fractionirt in zwei Portionen gefällt, nur die zweite in Salzsäure gelöst und das erhaltene Chlorbaryum noch weiter durch

\footnotetext{
${ }^{1}$ Bunsen, Pogg. Ann. Bd. 100, S. 85.
} 
wiederholtes Umkrystallisiren gereinigt. Um das Chlorstrontium möglichst rein zu gewinnen, wurde die käufliche Verbindung wiederholt aus Alkohol umkrystallisirt, fractionirt in zwei Portionen mit kohlensaurem Ammoniak gefällt, die zweite Fällung in Salpetersäure gelöst und das salpetersaure Salz durch Zusammenreiben und Auskochen mit Alkohol von den letzten Spuren Kalk befreit. Aus dem so gereinigten Producte wurde endlich durch Fällen mit kohlen- saurem Ammoniak und Auflösen des Niederschlags in Salzsäure das Chlorstrontium erhalten. Alle diese Reinigungen geschahen, so weit es ausführbar war, in Platingefässen.

In Fig. I Taf. VI [s. Abb. 1] ist der Apparat abgebildet, dessen wir uns meistens zur Beobachtung der Spectren bedient haben. $A$ ist ein innen ge-

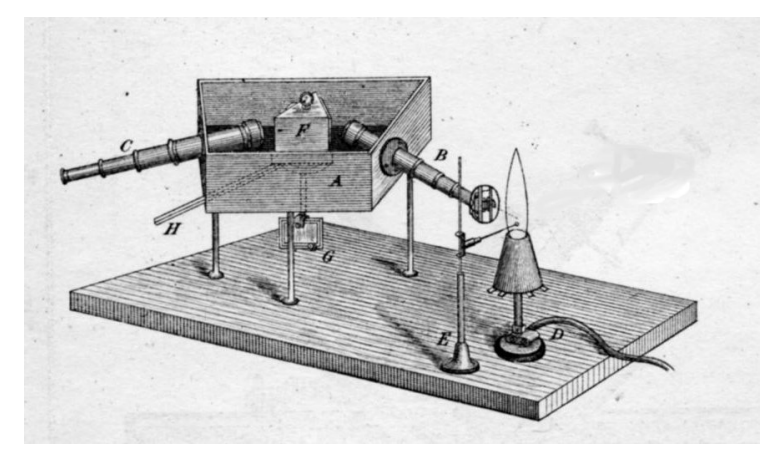

Abbildung 1:

schwärzter Kasten, dessen Boden die Gestalt eines Trapezes hat und der auf drei Füssen ruht; die beiden schiefen Seitenwände desselben, die einen Winkel etwa $58^{\circ}$ mit einander bilden, tragen die beiden kleinen Fernröhre $B$ und $C$. Die Ocularlinsen des ersteren sind entfernt und ersetzt durch eine Platte, in der ein aus zwei Messingschneiden gebildeter Spalt sich befindet, der in den Brennpunkt der Objectivlinse gestellt ist. Vor dem Spalte steht die Lampe $D$ so, dass der Saum ihrer Flamme von der Axe des Rohres $B$ getroffen wird. Etwas unterhalb der Stelle, wo die Axe den Saum trifft, taucht in denselben das zu einem kleinen Oehr gebogene Ende eines sehr feinen Platindrahtes, der von dem Träger $E$ gehalten wird; diesem Oehr ist eine Perle der zu untersuchenden, vorher entwässerten Chlorverbindung angeschmolzen. Zwischen den Objectiven der Fernröhre $B$ und $C$ steht ein Hohlprisma $F$ von $60^{\circ}$ brechendem Winkel, das mit Schwefelkohlenstoff angefüllt ist. Das Prisma ruht auf einer Messingplatte, die um eine verticale Axe drehbar ist. Diese Axe trägt an ihrem unteren Ende den Spiegel $G$ und darüber den Arm $H$, der als Handhabe dient, um das Prisma und den Spiegel zu drehen. Gegen den Spiegel ist ein kleines Fernrohr gerichtet, welches dem hindurchblickenden Auge das Spiegelbild einer in geringer Entfernung aufgestellten horizontalen 
Skale zeigt. Durch Drehung des Prismas kann man das ganze Spectrum der Flamme bei dem Verticalfaden des Fernrohrs $C$ vorbeiführen und jede Stelle des Spectrums mit diesem Faden zur Deckung bringen. Einer jeden Stelle des Spectrums entspricht eine an der Skale zu machende Ablesung. Ist das Spectrum sehr lichtschwach, so wird der Faden des Fernrohrs $C$ beleuchtet mit Hülfe einer Linse, die einen Theil der von einer Lampe ausgehenden Strahlen durch eine kleine Oeffnung wirft, die in der Ocularröhre des Fernrohrs $C$ seitlich angebracht ist.

Die Taf. V. [s. Abb. 2] dargestellten, mit Hülfe der oben erwähnten reinen Chlorverbindungen erzeugten Spectren haben wir mit denjenigen verglichen, welche man erhält, wenn man die Bromide, Iodide, Oxydhydrate, die schwefelsauren und kohlensauren Salze der entsprechenden Metalle in folgende Flammen bringt:

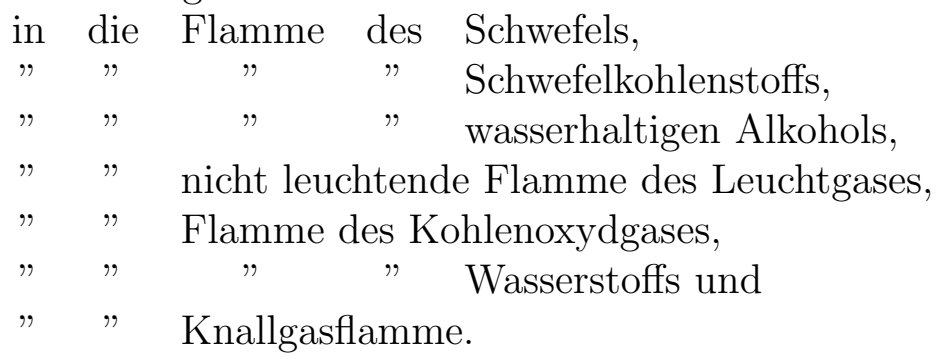

Bei dieser umfassenden und zeitraubenden Untersuchung, deren Einzelnheiten wir übergehen zu dürfen glauben, hat sich herausgestellt, dass die Verschiedenheit der Verbindungen, in denen die Metalle angewandt wurden, die Mannigfaltigkeit der chemischen Processe in den einzelnen Flammen und der ungeheuere Temperaturunterschied dieser letzteren keinen Einfluss auf die Lage der den einzelnen Metallen entsprechenden Spectrallinien ausübt.

Wie bedeutend die erwähnten Temperaturunterschiede sind, ergiebt sich aus der folgenden Betrachtung.

Man gelangt zu einer Schätzung der Temperatur einer Flamme mit Hülfe der Gleichung

$$
t=\sum g w / \sum p s
$$

in der $t$ die fragliche Temperatur der Flamme, $g$ das Gewicht eines der mit Sauerstoff verbrennenden Stoffe, $w$ die Verbrennungswärme desselben, $p$ das Gewicht und $s$ die specifische Wärme eines der Verbrennungsproducte bedeutet.

Nimmt man die Verbrennungswärme

$\begin{array}{cllc}\text { des } & \text { Schwefels } & \text { zu } & 2240^{\circ} \text { C. } \\ " & \text { Schwefelkohlenstoffs } & & 3400 \\ " & \text { Wasserstoffs } & " & 34462 \\ " & \text { Grubengases } & " & 13063\end{array}$


$\begin{array}{llrr}" & \text { Elayls } & " & 11640 \\ " & \text { Ditetryls } & " & 11529 \\ & \text { Kohlenoxyds } & & 2403\end{array}$

an und setzt nach REGNAULT die specifische Wärme bei constanten Druck

für schweflige Säure $=0,1553$

$"$ Kohlensäure $\quad=0,2184$

$"$ Stickstoff $\quad=0,2440$

$"$ Wasserdampf $=0,4750$,

so findet man hiernach die Temperatur

der Schwefelflamme $\quad 1820^{\circ} \mathrm{C}$.

" Schwefelkohlenstoffflamme 2195

" Leuchtgasflamme ${ }^{2} \quad 2350$

" Kohlenoxydflamme ${ }^{3} \quad 3042$

" Wasserstoffflamme in Luft ${ }^{4} 3259$

" Knallgasflamme ${ }^{5} \quad 8061$

Es zeigt sich, dass dieselbe Metallverbindung in einer dieser Flammen ein um so intensiveres Spectrum giebt, je höher die Temperatur derselben ist. Von den Verbindungen desselben Metalls liefert in einer Flamme diejenige die grössere Lichtstärke, der eine grössere Flüchtigkeit zukommt.

Um noch einen weiteren Beleg dafür zu erhalten, dass jedes der mehrfach genannten Metalle immer dieselben hellen Linien in dem Spectrum hervortreten lässt, haben wir die gezeichneten Spectren mit denjenigen verglichen, welche ein elektrischer Funke gewährt, der zwischen Elektroden, die aus jenen Metallen bestehen, überspringt.

Kleine Stücke von Kalium, Natrium, Lithium, Strontium und Calcium wurden an feine Platindrähte gebunden und in Glasröhren paarweise so eingeschmolzen, dass sie durch einen Zwischenraum von 1 bis $2 \mathrm{~mm}$ von einander getrennt waren und die Drähte die Glaswand durchdrangen. Jede dieser Röhren wurde vor dem Spalt des Spectralinstrumentes aufgestellt: mit Hülfe eines Ruhmkorf'schen Inductionsapparates liessen wir zwischen den genannten Metallstücken elektrische Funken überspringen und verglichen das Spectrum derselben mit dem Spectrum einer Gasflamme, in welche die Chlorverbindung des entsprechenden Metalls gebracht war. Die Flamme befand sich hinter der Glasröhre. Indem der Ruhmkorff'sche Apparat abwechselnd in und ausser Thätigkeit gesetzt wurde, war es leicht, ohne Messung sich mit Schärfe davon zu überzeugen, dass in dem glänzenden Spectrum des Funkens die hellen Linien des Flammenspectrums unverrückt vorhanden waren. Aus-

\footnotetext{
${ }^{2}$ Liebig's Ann. Bd. CXI, S. 258.

${ }^{3}$ Gasometrische Methode von R. Bunsen, S. 254.

${ }^{4}$ Ebendaselbst.

${ }^{5}$ Ebendaselbst.
} 
ser diesen traten in dem Funkenspectrum noch andere helle Linien auf, von denen ein Theil der Anwesenheit von fremden Metallen in den Elektroden, ein anderer dem Stickstoff, der die Röhren erfüllte, nachdem der Sauerstoff einen Theil der Elektroden oxydirt hatte, zugeschrieben werden muss. ${ }^{6}$

Es erscheint hiernach unzweifelhaft, dass die hellen Linien der gezeichneten Spectren als sichere Kennzeichen der Anwesenheit der betreffenden Metalle betrachtet werden dürfen. Sie können als Reactionsmittel dienen, durch welche diese Stoffe schärfer, schneller und in geringeren Mengen sich nachweisen lassen, als durch irgend ein anderes analytisches Hülfsmittel.

Die abgebildeten Spectren beziehen sich auf den Fall, dass der Spalt so weit ist, dass von den dunkeln Linien des Sonnenspectrums nur die deutlichsten wahrnehmbar sind, dass die Vergrösserung des Beobachtungs-Fernrohres eine geringe (etwa viermalige) und die Lichtstärke eine mässige ist. Diese Bedingungen scheinen uns die vortheilhaftesten, wenn es sich darum handelt, eine chemische Analyse durch Spectralbeobachtungen auszuführen. Der Anblick der Spectren kann unter anderen Bedingungen ein wesentlich anderer sein. Wird die Reinheit des Spectrums vermehrt, so zerfallen viele von den als einfach gezeichneten Linien in mehrere, die Natriumlinie z. B. in zwei; wird die Lichtstärke vermehrt, so zeigen sich in mehreren der gezeichneten Spectren neue Linien, und die Verhältnisse der Helligkeiten der alten werden andere. Im Allgemeinen wächst bei Vermehrung der Lichtstärke die Helligkeit einer dunkleren Linie schneller als die einer helleren, doch so, dass jene nicht diese überholt. Ein deutliches Beispiel hierfür bieten die beiden Lithiumlinien. Nur eine Ausnahme haben wir von dieser Regel beobachtet, und zwar bei der Linie $B a \eta$, welche bei geringer Lichtstärke gar nicht wahrnehmbar ist, während $B a \gamma$ sehr deutlich erscheint, und bei grosser Lichtstärke sehr viel heller als diese ist. Diese Thatsache scheint uns von Wichtigkeit, und wir werden dieselbe einer weiteren Untersuchung unterwerfen.

Es sollen jetzt die Eigentümlichkeiten der einzelnen Spectren, deren Kenntniss in praktischer Hinsicht von Wichtigkeit ist, näher besprochen, und die Vortheile, welche die auf sie gegründete chemisch-analytische Methode bietet, hervorgehoben werden.

\footnotetext{
${ }^{6}$ Als wir bei einem Versuche mit Strontiumelektroden ein mit Wasserstoff statt mit Stickstoff gefülltes Röhrchen anwandten, verwandelte sich der Funkenstrom sehr bald in einen Lichtbogen, während die Wände des Röhrchens sich mit einem grauen Beschlage bedeckten. Beim Oeffnen des Röhrchens unter Steinöl zeigte es sich, dass das Wasserstoffgas verschwunden und ein luftleerer Raum entstanden war. Das Gas scheint daher bei den ungeheuren Temperaturen des elektrischen Funkens das Strontiumoxyd, welches nicht völlig von der Oberfläche des Metalls entfernt worden war, reducirt zu haben.
} 


\section{Natrium.}

Von allen Spectralreactionen ist die des Natriums am empfindlichsten. Die gelbe Linie $\mathrm{Na} \alpha$, die einzige, welche das Natriumspectrum aufzuweisen hat, fällt mit der Fraunhofer'schen Linie $D$ zusammen und zeichnet sich durch ihre besonders scharfe Begrenzung und ihre ausserordentliche Helligkeit aus. Ist die Flammentemperatur sehr hoch und die Menge der angewandten Substanz sehr gross, so zeigen sich in den nächsten Umgebungen der Linie Spuren eines continuirlichen Spectrums. Schon an sich sehr schwache, in ihre Nähe fallende Linien anderer Stoffe erscheinen dann noch mehr geschwächt und werden daher nicht selten erst sichtbar, wenn die Natriumreaction zu erlöschen beginnt.

An der Sauerstoff-, Chlor-, Iod- und Brom-Verbindung, an dem schwefelsauren und kohlensauren Salze zeigt sich die Reaction am deutlichsten. Allein selbst bei den kieselsauren, borsauren, phosphorsauren und anderen feuerbeständigen Salzen fehlt sie nicht.

Schon Swan ${ }^{7}$ hat auf die Kleinheit der Kochsalzmengen aufmerksam gemacht, welche die Natriumlinie noch deutlich hervorbringen können.

Folgender Versuch zeigt, dass die Chemie keine einzige Reaction aufzuweisen hat, welche sich auch nur im Entferntesten mit dieser spectralanalytischen Bestimmung des Natriums an Empfindlichkeit vergleichen liesse. Wir verpufften in einer vom Standorte unseres Apparates möglichst entlegenen Ecke des Beobachtungszimmers, welches ungefähr 60 Kubikmeter Luft fasst, 3 Milligramm chlorsaures Natron mit Milchzucker, während die nicht leuchtende Lampe vor dem Spalt beobachtet wurde. Schon nach wenigen Minuten gab die allmälig sich fahlgelblich färbende Flamme eine starke Natriumlinie, welche erst nach 10 Minuten wieder völlig verschwunden war. Aus dem Gewichte des verpufften Natronsalzes und der im Zimmer enthaltenen Luft lässt sich leicht berechnen, dass in einem Gewichtstheile der letzteren nicht einmal 1/20000000 Gewichtstheil Natronrauch suspendirt sein konnte. Da sich die Reaction in der Zeit einer Secunde mit aller Bequemlichkeit beobachten lässt, in dieser Zeit aber nach dem Zufluss und der Zusammensetzung der Flammengase nur ungefähr $50 \mathrm{CC}$. oder 0,0647 Grm. Luft, welche weniger als 1/20000000 des Natronsalzes enthalten, in der Flamme zum Glühen gelangen, so ergiebt sich, dass das Auge noch weniger als 1/3000000 Milligramm des Natronsalzes mit der grössten Deutlichkeit zu erkennen vermag. Bei einer solchen Empfindlichkeit der Reaction wird es begreiflich, dass nur selten in glühender atmosphärischer Luft eine deutliche Natronreaction fehlt. Die Erde ist auf mehr als zwei Drittel ihrer Oberfläche mit einer Kochsalzlösung

\footnotetext{
${ }^{7}$ Pogg. Ann. Bd. C, S. 311.
} 
bedeckt, welche von den zu Schaumfällen sich überstürzenden Meereswogen unaufhörlich in Wasserstaub verwandelt wird. Die Meerwassertröpfchen, welche auf diese Art in die Atmosphäre gelangen, verdunsten und hinterlassen kochsalzhaltige Sonnenstäubchen, die zwar einen der Grösse nach wechselnden, aber wie es scheint nur selten fehlenden Gemengtheil der Atmosphäre ausmachen, und die vielleicht dazu bestimmt sind, den kleinen Organismen die Salze zuzuführen, welche die grösseren Pflanzeil und Thiere dem Boden entnehmen. Dieser durch Spectralanalyse leicht erweislichliche Kochsalzgehalt der Luft verdient noch in einer anderen Hinsicht Beachtung. Wenn es nämlich, wie man jetzt wohl kaum mehr bezweifeln kann, katalytische Einflüsse sind, welche die miasmatische Verbreitung der Krankheiten vermitteln, so möchte eine antiseptisch wirkende Substanz, wie das Kochsalz, selbst in verschwindend kleiner Menge wohl kaum ohne wesentlichen Einfluss auf solche Vorgänge in der Luft sein können. Aus täglichen, längere Zeit fortgesetzten Spectralbeobachtungen wird sich leicht erkennen lassen, ob die Intensitätsänderungen der durch die atmosphärischen Natriumverbindungen erzeugten Spectrallinie $\mathrm{Na} \alpha$ mit dem Erscheinen und mit der Verbreitungsrichtung endemischer Krankheiten in irgend einem Zusammenhange steht.

In der unerhörten Empfindlichkeit dieser Natronreaction ist zugleich der Grund zu suchen, dass alle der Luft ausgesetzten Gegenstände nach einiger Zeit bei dem Erhitzen in der Flamme die Natriumlinie zeigen, und dass es nur bei wenigen Verbindungen gelingt, selbst wenn man sie zehn- und mehrmal aus Wasser, das nur mit Platingefässen in Berührung kam, umkrystallisirt, die letzte Spur der Linie Na $\alpha$ zu beseitigen. Ein haarförmiger Platindraht, den man durch Ausglühen von jeder Spur Natron befreit hat, zeigt die Reaction auf das Deutlichste wieder, wenn man ihn einige Stunden der Luft ausgesetzt hat. Nicht minder zeigt sie der Staub, welcher sich in Zimmern aus der Luft absetzt, so dass z. B. das Abklopfen eines bestäubten Buches schon genügt, um in einer Entfernung von mehreren Schritten das heftigste Aufblitzen der $\mathrm{Na} \alpha$-Linie zu bewirken.

\section{Lithium.}

Der glühend leuchtende Dampf der Lithiumverbindungen giebt zwei scharf begrenzte Linien, eine gelbe sehr sehwache $\operatorname{Li} \beta$ und eine rothe, glänzende Linie $\operatorname{Li} \alpha$. An Sicherheit und Empfindlichkeit übertrifft auch diese Reaction alle in der analytischen Chemie bisher bekannten. Der Natriumreaction steht sie indessen an Empfindlichkeit etwas nach, vielleicht nur weil das Auge für gelbe Strahlen empfindlicher ist als für rothe. Durch Verpuffen von 9 Milligr. kohlensaurem Lithium mit einem grossen Ueberschuss von Milchzucker und 
chlorsaurem Kali in der ungefähr 60 Cubikmeter fassenden Luft des Zimmers war die Linie schon deutlich sichtbar. Das Auge kann daher auf diese Weise, wie eine, der oben angeführten ähnliche Rechnung zeigt, noch weniger als 9/1000000 eines Milligramms kohlensaures Lithium mit der grössten Schärfe erkennen. 0,05 Grm. desselben Salzes auf die erwähnte Art verpufft, ertheilte der Luft desselben Zimmers die Fähigkeit, länger als eine Stunde andauernd die $\operatorname{Li} \alpha$-Linie hervorzubringen.

Die Sauerstoff-, Chlor-, Jod- und Bromverbindung ist am geeignetsten zur Erkennung des Lithiums. Aber auch das kohlensaure, schwefelsaure und selbst das phosphorsaure Salz eignen sich fast ebenso gut zu diesem Zwecke. Lithionhaltige Fossilien, wie Triphyllin, Triphan, Petalit, Lepidolith, brauchen nur in die Flamme gehalten zu werden, um ohne weiteres die Linie Li $\alpha$ im intensivsten Glanze zu geben. Auf diese Weise lässt sich Lithion in manchen Feldspäthen, z. B. in Orthoklas von Baveno, unmittelbar nachweisen. Die Linie zeigt sich dann nur einige Augenblicke lang, gleich nach dem Einbringen der Probe in die Flamme. So zeigten sich als lithionhaltig die Glimmer von Altenberg und Penig, als frei von Lithium dagegen Glimmer von Miask, Aschaffenburg, Modum, Bengalen, Pennsylvanien etc. Wo in natürlich vorkommenden Silicaten nur ein verschwindend kleiner Lithiongehalt auftritt, entzieht sich derselbe der unmittelbaren Beobachtung. Die Prüfung geschieht dann in solchen Fällen am besten auf folgende Weise: man digerirt und verdampft eine kleine Menge der zu prüfenden Substanz mit Flusssäure oder Fluorammonium, dampft etwas Schwefelsäure über dem Rückstände ab und zieht die trockene Masse mit absolutem Alkohol aus. Die zur Trockenheit abgedampfte alkoholische Lösung wird dann noch einmal mit Alkohol extrahirt und die so erhaltene Flüssigkeit auf einer möglichst flachen Glasschale verdunstet. Der Anflug, welcher dabei zurückbleibt; lässt sich leicht mittels eines Radirmessers zusammenschaben und am Platindrähtchen in die Flamme bringen. 1/10 Milligr. davon reicht gewöhnlich für den Versuch vollkommen aus. Andere Verbindungen, als kieselsaure, in denen man noch die letzten Spuren Lithion entdecken will, werden nur durch Eindampfen mit Schwefelsäure oder auf irgend einem anderen Wege in schwefelsaure Salze verwandelt und dann ebenso behandelt.

Mit Hülfe dieses Verfahrens lässt sich leicht die unerwartete Thatsache ausser Zweifel setzen, dass das Lithion zu den am allgemeinsten in der Natur verbreiteten Stoffen gehört. Dasselbe liess sich mit der grössten Leichtigkeit schon in 40 Cubikcentimeter Meerwasser nachweisen, welches unter $39^{\circ}$ 14' westl. Länge und 41 $41^{\prime}$ nördl. Breite im Atlantischen Ocean geschöpft war. Asche von Fucoideen (Kelp), welche vom Golfstrom an die Schottischen Küsten getrieben werden enthielt erhebliche Spuren davon. Sämmtliche Orthoklase und Quarze aus dem Granit des Odenwaldes, die wir geprüft haben 
zeigten sich lithionhaltig. Ein sehr reines Trinkwasser aus einer Quelle am granitischen westlichen Abhänge des Neckarthales in Schlierbach bei Heidelberg enthielt Lithion, während die im bunten Sandstein entspringende Quelle, welche die Wasserleitung des hiesigen chemischen Laboratoriums speist, frei davon war. Mineralwasser, bei welchen Lithium kaum noch in 1 Liter nach dem gewöhnlichen analytischen Verfahren nachgewiesen werden kann, zeigt die $\operatorname{Li} \alpha$-Linie oft schon, wenn man nur einen Tropfen davon an einem Platindraht in die Flamme bringt ${ }^{8}$. Alle von uns untersuchten Odenwälder Aschen aus Hölzern, welche auf Granitboden wachsen, sowie Russische und andere käufliche Pottaschen enthalten Lithion. Selbst in den Aschen des Tabaks, der Weinblätter, des Rebholzes und der Weinbeeren ${ }^{9}$, sowie in der Asche der Feldfrüchte, welche in der Rheinebene bei Waghäusel, Deidesheim und Heidelberg auf nicht granitischem Boden gezogen werden, fehlt das Lithion eben so wenig, als in der Milch der Thiere, welche mit jenen Feldfrüchten genährt werden. ${ }^{10}$

Es braucht kaum bemerkt zu werden, dass ein Gemenge von flüchtigen Natron- und Lithionsalzen neben der Reaction des Natriums die des Lithiums mit einer kaum merklich verminderten Schärfe und Deutlichkeit zeigt. Die rothe Linie des letzteren erscheint durch eine kleine in die Flamme gebrachte Perle noch deutlich sichtbar, wenn diese Perle nur 1/1000 Lithiumsalz enthält, wobei das Auge für sich an der Flamme selbst nichts als das gelbe Licht des Natriums ohne jede Andeutung einer röthlichen Färbung wahrnimmt. In Folge der grösseren Flüchtigkeit der Lithionsalze hält die Natronreaction gewöhnlich etwas länger an. Wo es sich daher um die Erkennung sehr kleiner Spuren von Lithion neben Natron handelt, muss die Probeperle in die Flamme geschoben werden, während man schon durch das Fernrohr blickt. Man gewahrt dann die Lithiumlinie oft nur auf wenige Augenblicke unter den ersten Verflüchtigungsproducten.

Wo es sich bei der technischen Gewinnung der Lithiumverbindungen um die Auswahl des zu benutzenden Rohmaterials und die Auffindung einer zweckmässigen Darstellungsmethode handelt, gewährt die Spectralanalyse ein Hülfsmittel von unschätzbarem Werthe. So genügt es z. B. schon, von

\footnotetext{
${ }^{8}$ Wenn es sicb, darum handelt, eine Flüssigkeit in die Flamme zu bringen, so biegt man aus dem einen Ende eines pferdehaardicken Platindrahtes einen kleinen mit einem Durchmesser versehenen Ring und schlägt denselben platt. Lässt man in das so gebildete Oehr einen Flüssigkeitstropfen fallen, so bleibt eine für den Versuch hinreichende Menge darin hängen.

${ }^{9}$ In den bei der fabrikmässigen Weinsäurengewinnung fallenden Mutterlaugen concentrirt sich das Lithion so sehr, dass man aus denselben erhebliche Mengen davon darstellen kann.

${ }^{10}$ Herr Dr. Folwarezny hat sogar in der Asche des menschlichen Blutes und Muskelfleisches durch die Linie $\operatorname{Li} \alpha$ leicht Lithiumverbindungen nachweisen können.
} 
verschiedenen Soolmutterlaugen nur einen Tropfen in der Flamme zu verdampfen und durch das Fernrohr zu beobachten, um sich sogleich zu überzeugen, dass in vielen dieser Salinenrückstände ein reiches, bisher übersehenes Lithionmaterial gegeben ist. Dabei kann man im Verlaufe der Darstellung jeden Verlust an Lithion in den Nebenproducten und Abfallen duruch die Spectralreaction unmittelbar verfolgen und so leicht zweckmässigere Darstellungsmethoden als die bisher gebräuchlichen sich aufsuchen. ${ }^{11}$

\section{Kalium.}

Die flüchtigen Kaliumverbindungen geben in der Flamme ein sehr ausgedehntes continuirliches Spectrum, welches nur zwei charakteristische Linien zeigt; die eine Ka $\alpha$ in dem äussersten an die ultrarothen Strahlen grenzenden Roth, genau auf die dunkle Linie $A$ des Sonnenspectrums fallend, die andere Ka $\beta$ weit in Violet nach dem anderen Ende des Spectrums hin ebenfalls einer Fraunhofer'schen Linie entsprechend. Eine sehr schwache, mit der Fraunhofer'schen Linie $B$ zusammenfallende Linie, die ausserdem noch, aber nur bei der intensivsten Flamme, sichtbar wird, ist wenig charakteristisch. Die blaue Linie ist ziemlich schwach, eignet sich aber fast eben so gut wie die rothe Linie zur Erkennung des Kaliums. Die Lage beider Linien in der Nähe der beiden Grenzen der für das Auge wahrnehmbaren Strahlen macht die Reaction zu einer weniger empfindlichen. In der Luft unseres Zimmers wurde sie erst sichtbar, als wir gegen 1 Gramm mit Milchzucker gemengtes chlorsaures Kali abbrannten. Man kann daher dem Auge auf diese Weise nur ungefähr 1/1000 Milligr. chlorsaures Kali noch sichtbar machen.

Kalihydrat und sämmtliche Verbindungen des Kalis mit flüchtigen Säuren zeigen die Reaction ohne Ausnahme. Kalisilicate und ähnliche feuerbeständige Salze dagegen bringen sie für sich allein nur bei sehr vorwiegendem Kaligehalt hervor. Bei geringerem Kaligehalt darf man die Probeperle nur mit etwas kohlensaurem Natron zusammenschmelzen, um die charakteristischen Linien zum Vorschein zu bringen. Die Gegenwart von Natronsalzen verhindert mithin die Reaction nicht und beeinträchtigt die Empfindlichkeit derselben nur wenig. Orthoklas, Sanidin und Adular lassen sich dadurch leicht von Albit, Oligoklas, Labrador und Anorthit unterscheiden. Um verschwindend

\footnotetext{
${ }^{11}$ Wir erhielten nach einer solchen verbesserten Methode aus zwei Mineralwasserkrügen (gegen vier Liter) einer Soolmutterlauge, welche durch Eindampfen mit Schwefelsäure $1^{K}, 2$ Rückstand gaben, eine halbe Unze kohlensaures Lithion von der Reinheit des käuflichen, dessen Handelswerth ungefähr $140 \mathrm{fl}$. per Pfund beträgt. Eine grosse Zahl anderer Soolmutterlaugen, die wir untersuchten, zeigten einen ähnlichen Reichthum an Lithiumverbindungen.
} 
kleine Kalispuren noch nachzuweisen, braucht man die Silicate nur mit einem grosseu Ueberschuss von Fluorammonium auf einem Platindeckel schwach zu glühen und den Rückstand am Platindraht in die Flamme zu bringen. Auf diese Weise findet man, dass fast alle Silicate kalihaltig sind. Lithionsalze stören die Reaction eben so wenig. So genügt es z. B. schon, den Aschenstumpf einer Cigarre in die Flamme vor dem Spalt zu halten, um sogleich die gelbe Linie des Natriums und die beiden rothen des Kaliums und Lithiums, welches letztere Metall in den Tabaksaschen fast niemals fehlt, auf das Deutlichste hervorzubringen.

\section{Strontium.}

Die Spectren der alkalischen Erden stehen denen der Alkalien an Einfachheit bedeutend nach. Das des Strontiums ist besonders durch die Abwesenheit grüner Streifen charakterisirt. Acht Linien darin sind sehr ausgezeichnet, sechs rothe nämlich, eine orange und eine blaue. Die Orangelinie $S r \alpha$, welche dicht neben der Natriumlinie nach Roth hin auftritt, die beiden rothen Linien $\operatorname{Sr} \beta$, Sr $\gamma$ und endlich die blaue Linie $\operatorname{Sr} \delta$ sind ihrer Lage und Intensität nach die wichtigsten. Um die Empfindlichkeit der Reaction zu prüfen, erhitzten wir eine wässerige Chlorstrontiumlösung von bekanntem Salzgehalt in einem Platinschälchen rasch über einer grossen Flamme, bis das Wasser verdunstet war und die Schale zu glühen anfing. Hierbei decrepitirte das Salz zu mikroskopischen Partikelchen, die sich in Gestalt eines weissen Rauches in die Atmosphäre erhoben. Eine Wägung des Salzrückstandes in der Schale ergab, dass auf diese Weise 0,077 Grm, Chlorstrontium in Gestalt eines feinen Staubes in die 77000 Grm. wiegende Luft des Zimmers übergegangen war. Nachdem die Luft des Zimmers mittelst eines aufgespannten, rasch in Bewegung gesetzten Regenschirmes gleichmäßig durcheinander gemengt war, zeigten sich die charakteristischen Linien des Strontiumspectrums sehr schön ausgebildet. Man kann nach diesem Versuche die noch nachweisbare Chlorstroritiummenge zu 6/100000 eines Milligramms anschlagen.

Die Chlorverbindung und die übrigen Halogenverbindungen des Strontiums geben die Reaction am deutlichsten. Strontianerdehydrat und kohlensaure Strontianerde zeigen sie viel schwächer; schwefelsaure noch schwächer; die Verbindungen mit feuerbeständigen Säuren am schwächsten, oder gar nicht. Man bringt daher die Probeperle zunächst für sich und dann nach vorgängiger Befeuchtung mit Salzsäure in die Flamme. Hat man Schwefelsäure in der Perle vorauszusetzen, so hält man sie vor dem Befeuchten mit Salzsäure einige Augenblicke in den reducirenden Theil der Flamme, um das schwefelsaure Salz in die durch Chlorwasserstoffsäure zersetzbare Schwefelverbindung um- 
zuändern. Zur Erkennung des Strontiums in Verbindungen mit Kieselsäure, Phosphorsäure, Borsäure oder anderen feuerbeständigen Säuren verfährt man am besten auf folgende Weise: Zum Aufschliessen der Probe mit kohlensaurem Natron dient, statt eines Platintiegels, eine conische Spirale von Platindraht. Dieselbe wird in der Flamme weissglühend gemacht und in entwässertes, fein pulverisirtes, lockeres kohlensaures Natron getaucht, welches wo möglich noch so viel Wasser enthält, dass die nöthige Menge des Salzes schon bei dem ersten Eintauchen daran hängen bleibt. In dieser Spirale lässt sich die Schmelzung viel schneller als in einem Platintiegel bewerkstelligen, da die zu erhitzende Masse des Platins nur gering ist und das zu schmelzende Salz mit der Flamme in unmittelbare Berührung kommt. Hat man die aufzuschliessende, fein pulverisirte Substanz mittelst einer kleinen Platinschaufel in die glühend flüssige Soda eingetragen und einige Minuten im Glühen erhalten, so braucht man die mit ihrer Spitze nach oben gekehrte Spirale nur auf den Rand des Lampentellers aufzuklopfen, um den Inhalt derselben in Gestalt einer grossen erkaltenden Kugel auf dem Teller zu erhalten. Man bedeckt die Kugel mit einem Blättchen Schreibpapier und zerdrückt dieselbe mittelst einer elastischen Messerklinge, die man auch nach Entfernung des Papiers benutzt, um die Masse weiter noch zum feinsten Pulver zu zerdrücken. Dieses wird an den Rand des etwas abwärts geneigten Tellers zusammengehäuft, vorsichtig mit heissem Wasser übergossen, das man durch sanftes Hin- und Herneigen des Tellers über der aufgehäuften Substanz hin und her fliessen lässt, und endlich die über dem Bodensatz stehende Flüssigkeit abdekantirt. Es gelingt leicht, unter abwechselndem Erwärmen des Tellers durch mehrmalige Wiederholung dieser Operation die löslichen Salze auszuziehen, ohne den Bodensatz aufzurühren und erhebliche Mengen davon zu verlieren. Wendet man statt des Wassers eine Kochsalzlösung an, so gelingt die Operation noch leichter und sicherer. Der Rückstand enthält das Strontium als kohlensaures Salz, von dem schon einige Zehntel Milligramm am Platindraht mit etwas Salzsäure befeuchtet die intensivste Reaction geben. Es wird auf diese Art möglich, ohne Platintiegel, ohne Reibschale, ohne Digerirschale und ohne Trichter und Filter alle erforderlichen Operationen des Aufschliessens, Zerkleinerns, Digerirens und Auswaschens in wenigen Minuten auszuführen.

Die Reaction des Kaliums und Natriums wird durch die Gegenwart des Strontiums nicht gestört. Auch die Lithiumreaction tritt neben den drei erwähnten in voller Deutlichkeit auf, wenn die Lithiummenge gegen die des Strontiums nicht zu gering ist. Die Lithiumlinie $\mathrm{Li} \alpha$ erscheint dann als ein schmaler, intensiv rother, scharf begrenzter Streifen auf dem schwächer rothen Grunde des breiten Strontiumstreifens $\operatorname{Sr} \beta$. 


\section{Calcium.}

Das Calciumspectrum lässt sich schon auf den ersten Blick von den vier bisher betrachteten Spectren daran unterscheiden, dass es in Grün eine höchst charakteristische und intensive Linie, $\mathrm{Ca} \beta$, enthält. Als zweites nicht minder charakteristisches Kennzeichen kann die ebenfalls sehr intensive Orangelinie Ca $\alpha$ dienen, welche erheblich weiter nach dem rothen Ende des Spectrums hin liegt als die Natronlinie $\mathrm{Na} \alpha$ und die Orangelinie des Strontiums $\operatorname{Sr} \alpha$, Durch Abbrennen eines Gemenges von Chlorcalcium, chlorsaurem Kali und Milchzucker erhält man einen Rauch, dessen Reaction ungefähr von gleicher Empfindlichkeit ist mit dem unter denselben Verhältnissen hervorgebrachten Chlorstrontiumrauch. Aus einem auf diese Weise an gestellten Versuche ergab sich, dass 6/1000000 Milligramm Chlorcalcium noch leicht und mit völliger Sicherheit erkannt werden können. Nur die in der Flamme flüchtigen Calciumverbindungen zeigen die Reaction, und zwar mit um so grösserer Deutlichkeit, je flüchtiger sie sind. Chlorcalcium, Jodcalcium Bromcalcium stehen in dieser Beziehung oben an. Schwefelsaurer Kalk giebt das Spectrum erst, nachdem er angefangen hat basisch zu werden, dann aber sehr glänzend und lange andauernd. Ebenso entwickelt sich die Reaction des kohlensauren Kalks am deutlichsten, nachdem die Kohlensäure entwichen ist.

Verbindungen des Calciums mit feuerbeständigen Säuren verhalten sich in der Flamme indifferent; werden sie durch Chlorwasserstoffsäure angegriffen, so lässt sich die Reaction einfach auf folgende Weise erhalten: Man bringt einige Milligramme oder selbst nur einige Zehntel Milligramme der fein pulverisirten Substanz an das etwas befeuchtete plattgeschlagene Platinöhr in den wenig heissen Theil der Flamme, bis das Pulver ohne zu schmelzen angefrittet ist. Lässt man einen Tropfen Salzsäure in das Oehr fallen, so bleibt derselbe zum grössten Theil darin hängen. Schiebt man diesen Tropfen vor dem Spalt des Spectralapparates in den heissesten Theil der Flamme, so verdampft er, und zwar in Folge des Leidenfrost'schen Phänomens, ohne ins Kochen zu gerathen. Blickt man, während der Tropfen verdampft, durch das Fernrohr, so erscheint in dem Augenblick, wo die letzten Antheile der Flüssigkeit in Dampf verwandelt werden, ein glänzendes Calciumspectrum, welches bei geringem Kalkgehalt nur einen Moment aufblitzt, bei erheblicheren Kalkmengen aber mehr oder weniger lange anhält.

Nur in Silicaten, welche von Salzsäure angegriffen werden, lässt sich der Kalk auf diese Weise finden; in nicht durch Salzsäure angreifbaren Silicaten gelingt die Nachweisung am besten folgendermassen: Einige Milligramm der zu prüfenden, auf das Feinste pulverisirten Substanz werden auf einem flachen Tiegeldeckel von Platin mit ungefähr einem Gramm halb zerflossenem Fluorammonium versetzt und der Deckel in die Flamme gehalten, 
bis er nach Verflüchtigung des Fluorammoniums glüht. Man befeuchtet den auf dem Deckel befindlichen Salzanflug mit 1 bis 2 Tropfen Schwefelsäure, und entfernt den Ueberschuss derselben durch abermaliges Erhitzen über der Flamme. Wird der jetzt aus schwefelsauren Salzen bestehende Anflug auf dem Deckel mit dem Fingernagel oder einem Spatelchen zusammengeschabt und ungefähr ein Milligramm davon mittelst des Drahtes in die Flamme gebracht, so erhält man, wenn $\mathrm{Ka}, \mathrm{Na}$ und $\mathrm{Li}$ vorhanden sind, zunächst die charakteristischen Reactionen dieser drei Körper neben oder nach einander. Ist noch Kalk und Strontian vorhanden, so erscheinen deren Spectren gewöhnlich erst etwas später, nachdem das $\mathrm{Ka}, \mathrm{Na}$ und $\mathrm{Li}$ verflüchtigt ist. Bei sehr geringem Calcium- oder Strontiuingehalt bleibt die Reaction dieser Metalle aus; man erhält sie dann aber sogleich, wenn man den im Reductionsraum der Flamme einige Augenblicke behandelten Draht mit Salzsäure betropft und wieder in die Flamme bringt.

Alle diese Proben, die Erhitzung für sich oder mit Salzsäure, die Behandlung mit Fluorammonium für sich oder mit Schwefelsäure und Salzsäure geben dem Mineralogen und mehr noch dem Geognosten eine Reihe höchst einfacher Kennzeichen an die Hand, um viele in der Natur auftretende Substanzen, und namentlich die einander so ähnlichen aus kalkhaltigen Doppelsilicaten bestehenden Mineralien noch in den kleinsten Splitterchen mit einer Sicherheit zu bestimmen, wie sie sonst kaum bei einem reichlich zu Gebote stehenden Material durch weitläuftige und zeitraubende Analysen erreichbar ist. Einige Beispiele werden dies am besten zeigen.

1. Ein Tropfen Meerwasser am Platindraht verflüchtigt zeigt eine starke Natriumreaction, und nach Verflüchtigung des Kochsalzes eine schwache Calciumreaction, die durch Befeuchten des Drahtes mit Salzsäure auf Augenblicke höchst intensiv wird. Behandelt man einige Decigramme Meerwasserrückstand auf die beim Lithium angegebene Weise mit Schwefelsäure und Alkohol, so erhält man leicht die Reaction des Kaliums und Lithiums. Die Gegenwart des Strontiums im Meerwasser kann am besten in den Kesselsteinen der Seedampfschiffe nachgewiesen werden. Die filtrirte salzsaure Lösung desselben hinterlässt nach dem Abdampfen und Auflösen in möglichst wenig Alkohol eine von basischem Eisenchlorid gelblich gefärbte Trübung, die sich nach einigen Tagen absetzt und auf einem Filterchen gesammelt und mit Alkohol ausgewaschen werden kann. Das in einem feinen Platindraht verbrannte Filter giebt neben den Calciumlinien ein vollständiges und intensives Strontiumspectrum.

2. Soolwasser zeigen oft schon unmittelbar die Kalium-, Natrium-, Lithium-, Calcium- und Strontiumreaction. Bringt man z. B. einen Tropfen des Dürkheimer oder Kreuznacher Mineralwassers in die Flamme, so erhält man die Linien $\mathrm{Na} \alpha, \mathrm{Li} \alpha, \mathrm{Ca} \alpha$ und $\mathrm{Ca} \beta$. Wendet man statt des Soolwassers 
einen Tropfen seiner Mutterlauge an, so entstehen dieselben Linien mit dem intensivsten Glanze. In dem Maasse als das Chlornatrium und Chlorlithium verdampft und das Chlorcalcium basischer wird, entwickeln sich allmählich die charakteristischen Linien des Strontiumspectrums, welches sich nach und nach immer glänzender in seiner ganzen Vollständigkeit zeigt. Man erhält hier also durch den blossen Anblick eines einzigen in der Flamme verflüchtigten Tropfens in wenigen Augenblicken die vollständige Analyse eines Gemenges von fünf Stoffen.

3. Der Aschenstumpf einer Cigarre mit etwas $\mathrm{HCl}$ befeuchtet und in die Flamme gehalten, giebt die Linien $\mathrm{Na} \alpha, \operatorname{Ka} \alpha, \operatorname{Li} \alpha, \mathrm{Ca} \alpha, \mathrm{Ca} \beta$.

4. Kaliglas von einer Verbrennungsröhre gab sowohl mit als ohne Salzsäure $\mathrm{Na} \alpha$ und $\mathrm{Ka} \alpha$, mit Fluorammonium und Schwefelsäure behandelt noch $\mathrm{Ca}$ $\alpha, C a \beta$ und Spuren von $\operatorname{Li} \alpha$.

5. Orthoklas von Baveno giebt für sich oder mit Salzsäure nur Na $\alpha$ nebst Spuren von Ka $\alpha$ und $\mathrm{Li} \alpha$, mit Fluorammonium und Schwefelsäure die intensiven Linien $\mathrm{Na} \alpha$, Ka $\alpha$ und etwas schwächer $\mathrm{Li} \alpha$. Nach Verflüchtigung der so nachgewiesenen Bestandteile, mit $\mathrm{HCl}$ in die Flamme gebracht, giebt die Probe nur ein kaum unterscheidbares Aufblitzen der Linien Ca $\alpha$ und Ca $\beta$. Der nach diesen Prüfungen dem Platindrahte angefrittete Rückstand zeigt, mit salpetersaurem Kobaltoxydul befeuchtet und geglüht, die für Thonerde charakteristische Färbung. Nimmt man noch die bekannte Reaction auf Kieselerde hinzu, so ergiebt sich aus diesen in wenigen Minuten aus führbaren Prüfungen, dass der Orthoklas von Baveno Kieselerde, Thonerde, Kali mit Spuren von Natron, Kalkerde und Lithion enthält, so wie dass jede Spur von Baryterde und Strontianerde darin fehlt.

6. Adular vom Gotthard verhielt sich ganz ähnlich wie der Orthoklas von Baveno, nur dass die Lithiumreaction völlig, die Calciumreaction fast völlig fehlte.

7. Labradorit von St. Paul giebt für sich nur die Natriumlinie Na $\alpha$, nicht aber das Calciumspectrum. Die mit Chlorwasserstoffsäure befeuchtete Probe aber bringt die Calciumlinien $\mathrm{Ca} \alpha$ und $\mathrm{Ca} \beta$ sehr glänzend hervor. Bei der Probe mit Fluorammonium erhält man noch eine schwache Kaliumreaction und kaum bemerkbare Spuren von Lithium.

8. Labradorit aus dem Kugeldiorit von Corsika verhielt sich ebenso, nur dass die Spuren der Lithiumreaction fehlten.

9. Mosandrit aus Brevig und Tscheffkinit aus dem Ilmengebirge gaben für sich nur die Natriumreaction, bei der Behandlung mit Salzsäure aber die Calciumlinien $\mathrm{Ca} \alpha$ und $\mathrm{Ca} \beta$.

10. Melinophan von Lamoe gab für sich nur $\mathrm{Na} \alpha$, mit Salzsäure aber noch $C a \alpha, C a \beta$ und $\operatorname{Li} \alpha$.

11. Scheelit und Sphen geben schon bei Behandlung mit Salzsäure die 
Oalciumreaction sehr intensiv.

12. Finden sich geringe Mengen Strontium neben dem Calcium, so wählt man am zweckmässigsten die Linie $\operatorname{Sr} \delta$ zur Erkennung der ersteren. Mit Hülfe derselben gelingt es leicht, in sehr vielen neptunischen Kalksteinen einen geringen Strontiumgehalt nachzuweisen. $\mathrm{Na} \alpha, \operatorname{Li} \alpha$, Ka $\alpha$, besonders Li $\alpha$, zeigen sich schon unmittelbar bei dem Glühen des Kalksteins in der Flamme. Durch Salzsäure in Chlorcalcium verwandelt und in dieser Form in die Flamme gebracht, geben diese Gesteine dieselben Linien und ausserdem häufig noch deutlich genug die Linie $S r \delta$. Dieselbe erscheint aber nur auf kürzere Zeit, indem sie sich in Folge der Verdampfungsprocesse in der Flamme allmählich entwickelt und kurz vor dem Erblassen des Kalkspectrums am deutlichsten hervorzutreten pflegt.

Auf diesem Wege wurden die Linien $\mathrm{Na} \alpha, \mathrm{Li} \alpha, \mathrm{Ka} \alpha, \mathrm{Ca} \alpha, \mathrm{Ca} \beta, \operatorname{Sr} \delta$ bei folgenden Kalksteinen gefunden:

Silurkalk ${ }^{12}$ von Kugelbad bei Prag,

Wellenkalk (Muschelkalk) von Rohrbach bei Heidelberg,

Liaskalk von Maisch in Baden,

Kreide aus England.

Folgende Kalksteine zeigten nur die Linien $\mathrm{Na} \alpha, \mathrm{Li} \alpha, \mathrm{Ka} \alpha, \mathrm{Ca} \alpha, \mathrm{Ca}$ $\beta$, ohne die blaue Strontiumlinie:

Marmor von Auerbach aus dem Granit ${ }^{13}$,

Devonkalk von Gerolstein in der Eifel,

Kohlenkalk von Planitz in Sachsen,

Zechstein von Nordhausen am Harz,

Jurakalk vom Streitberg in Franken.

Man sieht schon aus diesen wenigen Versuchen, dass umfassendere und sorgfältige spectralanalytische Untersuchungen über den Lithium-, Kalium-, Natrium- und Strontiumgehalt verschiedener Kalkbildungen mit Beziehung auf die Altersfolge und locale Verbreitung derselben von grossem geologischen Interesse sind, und vielleicht zu unerwarteten Aufschlüssen über die Natur der früheren Oceane und Meeresbecken, in welchen die Bildung jener Kalkgebirge erfolgte, führen können.

\footnotetext{
${ }^{12}$ Die Lithiumlinie war bei dieser Gebirgsart nicht mit Sicherheit zu erkennen, die Linie Sr $\delta$ dagegen sehr stark.

${ }^{13}$ Mittelst des oben beschriebenen Verfahrens mit Alkohol wurde aus 20 Grm. dieses Marmors so viel salpetersaurer Strontian erhalten, dass sich damit ein vollständiges intensives Strontiumspectrum hervorbringen liess. Ob sich auch die übrigen aufgeführten Kalksteine, auf diese Art behandelt, als strontiumhaltig erweisen, haben wir nicht untersucht.
} 


\section{Baryum.}

Das Baryumspectrum ist das verwickeltste unter den Spectren der Metalle der Alkalien und alkalischen Erden. Von den bisher betrachteten unterscheidet es sich schon auf den ersten Blick durch die grünen Linien Ba $\alpha$ und $\mathrm{Ba} \beta$, welche alle übrigen an Intensität übertreffen und bei schwacher Reaction zuerst erscheinen und zuletzt wieder verschwinden. $\mathrm{Ba} \gamma$ ist weniger empfindlich, aber immer noch als charakteristische Linie zu betrachten. Die verhältnissmässig ziemlich grosse Ausdehnung des Spectrums ist Ursache, dass überhaupt die Spectralreaction der Baryumverbindungen etwas weniger empfindlich ist als die der bisher betrachteten Körper. 0,3 Grm. chlorsaurer Baryt mit Milchzucker gaben in unserem Zimmer verbrannt, nachdem die Luft vermittelst eines aufgespannten Regenschirmes gehörig durchgemengt war, längere Zeit auf das deutlichste die Linie $B a \alpha$. Man kann daher aus einer der beim Natrium ausgeführten ähnlichen Rechnung schliessen, dass durch die Reaction noch weniger als ungefähr 1/1000 Milligramm mit völliger Deutlichkeit angezeigt wird.

Chlorbaryum, Brombaryum, Jodbaryum, Fluorbaryum, Baryterdehydrat, kohlensaurer und schwefelsaurer Baryt zeigen die Reaction am ausgezeichnetsten, und können daher durch unmittelbares Erhitzen in der Flamme erkannt werden.

Durch Salzsäure angreifbare, Baryterde enthaltende Silicate geben die Reaction, wenn sie, wie beim Kalk angegeben, mit einem Tropfen Salzsäure in die Flamme gebracht werden, ebenfalls. So erzeugt z. B. Barytharmotom, auf diese Weise behandelt, die Linie $\mathrm{Ca} \alpha$ und $\mathrm{Ca} \beta$ neben den Linien $\mathrm{Ba} \alpha$ und $B a \beta$.

Verbindungen der Baryterde mit feuerbeständigen Säuren, die sich mit und ohne Salzsäure in der Flamme indifferent verhalten, schliesst man am besten auf die beim Strontium angegebene Weise mit kohlensaurem Natron auf und prüft den dadurch erhaltenen kohlensauren Baryt. Kommen in solchen Verbindungen $\mathrm{Ca}$, Ba und $\mathrm{Sr}$ in sehr ungleichen Mengen gemeinschaftlich vor, so löst man die durch Aufschliessen erhaltenen kohlensauren Salze in einem Tropfen Salpetersäure und zieht aus dem abgedampften Rückstand den Kalk durch Alkohol aus. Der Rückstand enthält dann noch Baryt und Strontium, die sich, wenn sie nicht in allzu ungleicher Menge vorkommen, leicht neben einander erkennen lassen. Handelt es sich darum, die letzten noch wahrnehmbaren, Spuren von $\mathrm{Sr}$ oder Ba nachzuweisen, so verwandelt man den Rückstand durch Glühen mit Salmiak in Chlorverbindungen, aus denen sich das Chlorstrontium durch Alkohol in der zur Erkennung hinlänglich concentrirten Form leicht ausziehen lässt. Sind unter den nachzuweisenden Stoffen nicht einzelne in verschwindend kleinen Mengen vorhanden, 
so werden alle solche vorgängige Scheidungen ganz unnöthig, wie folgender Versuch zeigt: Ein Gemenge von Chlornatrium, Chlorkalium, Chlorlithium, Chlorcalcium, Chlorstrontium und Chlorbaryum, welches von jedem dieser sechs Stoffe höchstens 1/10 Milligramm enthielt wurde in die Flamme gebracht und beobachtet. Zuerst erschien die intensiv gelbe Natronlinie $\mathrm{Na} \alpha$ auf dem Untergrunde eines schwachen continuirlichen Spectrums. In dem Maasse, als dieses zu erblassen begann, entwickelte sich die scharf begrenzte intensiv rothe Linie des Lithiums $\mathrm{Li} \alpha$ und jenseits derselben, noch weiter von der Natriumlinie entfernt die mattere Kaliumlinie Ka $\alpha$, indess die Baryumlinien $\mathrm{Ba} \alpha$ und $\mathrm{Ba} \beta$ in ihrer charakteristischen Lage und eigenthümlichen Schattirung auf das Deutlichste hervortraten. Indem sich darauf die Verbindungen des Kaliums, Lithiums und Baryums nach und nach verflüchtigten, erblassten oder verschwanden ihre Linien wieder allmählich der Reihe nach, bis sich nach einigen Minuten aus den immer weniger überlagerten Linien des Calciums und Strontiums, wie aus einem Nebelbilde, die Linien Ca $\alpha$, Ca $\beta$ und $\operatorname{Sr} \alpha, \operatorname{Sr} \beta, \operatorname{Sr} \gamma$ und $\operatorname{Sr} \delta$ mit aller Schärfe in ihrer charakteristischen Form, Schattirung und Lage hervorhoben, um dann erst nach sehr langer Zeit wieder zu erblassen und gänzlich zu verschwinden.

Die Abwesenheit irgend eines oder mehrerer dieser Gemengtheile giebt sich bei diesen Beobachtungen augenblicklich durch die Abwesenheit der ihnen zugehörigen Linien zu erkennen.

Für Denjenigen, welcher die einzelnen Spectren aus wiederholter Anschauung kennt, bedarf es einer genauen Messung, der einzelnen Linien nicht; ihre Farbe, ihre gegenseitige Lage, ihre eigenthümliche Gestalt und Abschattirung, die Abstufungen ihres Glanzes sind Kennzeichen, welche selbst für den Ungeübten zur sicheren Orientirung vollkommen hinreichen. Diese Kennzeichen sind den Unterscheidungsmerkmalen zu vergleichen, welche wir bei den als Reactionsmittel benutzten, ihrem äusseren Ansehen nach höchst verschiedenartigen Niederschlägen antreffen. Wie es als Charakter einer Fällung gilt, dass sie gelatinös, pulverförmig, käsig, körnig oder krystallinisch ist, so zeigen auch die Spectrallinien ihr eigenthümliches Verhalten, indem die einen an ihren Rändern scharf begrenzt, die anderen, entweder nur nach einer oder nach beiden Seiten entweder gleichartig oder ungleichartig verwaschen, oder indem die einen breiter, die anderen schmäler erscheinen. Und wie wir nur diejenigen Niederschlage, welche bei möglichst grosser Verdünnung der zu fällenden Substanz noch zum Vorschein kommen, als Erkennungsmittel verwenden, so benutzt man auch in der Spectralanalyse zu diesem Zwecke nur diejenigen Linien, welche zu ihrer Erzeugung die geringste Menge Substanz und 
eine nicht allzu hohe Temperatur erfordern. In Beziehung auf solche Kennzeichen stehen sich daher beide Methoden ziemlich gleich. Dagegen gewährt die Spectralanalyse rücksichtlich der als Reactionsmittel benutzten Farbenerscheinungen eine Eigentümlichkeit, die ihr unbedingt einen Vorzug vor jeder anderen analytischen Methode sichern muss. Unter den Niederschlägen, die zur Erkennung von Stoffen bestimmt sind, erscheinen die meisten weiss und nur einige gefärbt. Dabei ist die Färbung der letzteren nur wenig constant und variirt in den verschiedensten Abstufungen, je nach der dichteren oder mehr zertheilten Form der Fällung. Oft reicht schon die kleinste Beimengung eines fremden Stoffes hin, eine charakteristische Färbung bis zur Unkenntlichkeit zu verwischen. Feinere Farbenunterschiede der Niederschläge kommen daher als chemische Kennzeichen gar nicht mehr in Frage. Bei der Spectralanalyse dagegen erscheinen die farbigen Streifen unberührt von solchen fremden Einflüssen und unverändert durch die Dazwischenkunft anderer Stoffe. Die Stellen, welche sie im Spectrum einnehmen, bedingen eine chemische Eigenschaft, die so unwandelbarer und fundamentaler Natur ist, wie das Atomgewicht der Stoffe, und lassen sich daher mit einer fast astronomischen Genauigkeit bestimmen. Was aber der spectralanalytischen Methode eine ganz besondere Bedeutung verleiht, ist der Umstand, dass sie die Schranken, bis zu welchen bisher die chemischen Kennzeichen der Materie reichten, fast ins Unbegrenzte hinausrückt. Sie verspricht uns über die Verbreitung und Anordnung der Stoffe in den geologischen Formationen die werthvollsten Aufschlüsse. Schon die wenigen Versuche, welche diese Abhandlung enthält, führen zu dem unerwarteten Aufschlusse, dass nicht nur Kalium, und Natrium, sondern auch Lithium und Strontium zu den zwar nur in geringer Menge, aber allgemein verbreiteten Stoffen unseres Erdkörpers gezählt werden müssen.

Für die Entdeckung bisher noch nicht aufgefundener Elemente dürfte die Spectralanalyse eine nicht minder wichtige Bedeutung gewinnen. Denn wenn es Stoffe giebt, die so sparsam in der Natur verbreitet sind, dass uns die bisherigen Mittel der Analyse bei ihrer Erkennung und Abscheidung im Stiche lassen, so wird man hoffen dürfen, viele solcher Stoffe durch die einfache Betrachtung ihrer Flammenspectren noch in Mengen zu erkennen und zu bestimmen, die sich auf gewöhnlichem Wege jeder chemischen Wahrnehmung entziehen. Dass es wirklich solche bisher unbekannte Elemente giebt, davon haben wir uns bereits zu überzeugen Gelegenheit gehabt. Wir glauben, auf unzweifelhafte Resultate der spectralanalytischen Methode gestützt, mit völliger Sicherheit schon jetzt die Behauptung aufstellen zu können, dass es neben dem Kalium, Natrium und Lithium noch ein viertes der Alkaliengruppe angehöriges Metall giebt, welches ein eben so charakteristisches und einfaches Spectrum giebt wie das Lithium - ein Metall, das mit unserem Spectralapparate nur zwei Linien zeigt, eine schwache blaue, die mit 
der Strontiumlinie $\operatorname{Sr} \delta$ fast zusammenfällt, und eine andere blaue, die nur um Weniges weiter nach dem violetten Ende des Spectrums hin liegt und an Intensität und Schärfe der Begrenzung mit der Lithiumlinie wetteifert.

Bietet einerseits die Spectralanalyse, wie wir im Vorstehenden gezeigt zu haben glauben, ein Mittel von bewunderungswürdiger Einfachheit dar, die kleinsten Spuren gewisser Elemente in irdischen Körpern zu entdecken, so eröffnet sie andererseits der chemischen Forschung ein bisher völlig verschlossenes Gebiet, das weit über die Grenzen der Erde, ja selbst unseres Sonnensystems, hinausreicht. Da es bei der in Rede stehenden analytischen Methode ausreicht, das glühende Gas, um dessen Analyse es sich handelt, zu sehen, so liegt der Gedanke nahe, dass dieselbe auch anwendbar sei auf die Atmosphäre der Sonne und der helleren Fixsterne. Sie bedarf aber hier einer Modification wegen des Lichtes, welches die Kerne dieser Weltkörper ausstrahlen. In seiner Abhandlung: "über das Verhältniss zwischen dem Emissionsvermögen und dem. Absorptionsvermögen der Körper für Wärme und Licht" ${ }^{14}$ hat Einer von uns durch theoretische Betrachtungen nachgewiesen, dass das Spectrum eines glühenden Gases umgekehrt wird, d. h. dass die hellen Linien in dunkele sich verwandeln, wenn hinter dasselbe eine Lichtquelle von hinreichender Intensität gebracht wird, die an sich ein continuirliches Spectrum giebt. Es lässt sich hieraus schliessen, dass das Sonnenspectrum mit seinen dunkeln Linien nichts Anderes ist, als die Umkehrung des Spectrums, welches die Atmosphäre der Sonne für sich zeigen würde. Hiernach erfordert die chemische Analyse der Sonnenatmosphäre nur die Aufsuchung derjenigen Stoffe, die, in eine Flamme gebracht, helle Linien hervortreten lassen, die mit den dunkeln Linien des Sonnenspectrums coincidiren.

An dem angeführten Orte sind als experimentelle Belege für den erwähnten theoretisch abgeleiteten Satz die folgenden Versuche angeführt:

Die helle rothe Linie im Spectrum einer Gasflamme, in die eine Perle von Chlorlithium gebracht ist, verwandelt sich in eine schwarze, wenn man volles Sonnenlicht durch die Flamme gehen lässt.

Ersetzt man die Perle von Chlorlithium durch eine von Chlornatrium, so zeigt sich im Sonnenspectrum die dunkle Doppellinie $D$ (die mit der hellen Natriumlinie coincidirt) in ungewöhnlicher Deutlichkeit.

In dem Spectrum des Drumond'schen Lichtes tritt die dunkle Doppellinie $D$ auf, wenn man seine Strahlen durch die Flamme von wässerigem Alkohol gehen lässt, in den man Chlornatrium gebracht hat. ${ }^{15}$

\footnotetext{
${ }^{14}$ Kirchhoff, Pogg. Ann., Bd. CIX, S. 275. Oben S. 570.

${ }^{15}$ In der Märznummer des Phil. Mag. für 1860 erinnert Stokes daran, dass FoucAulT schon im Jahre 1849 eine Beobachtung gemacht hat, die der oben erwähnten ähnlich ist. Bei der Untersuchung des elektrischen Bogens zwischen Kohlenspitzen bemerkte dieser (l'Institut 1849 p. 45), dass in dem Spectrum desselben helle Linien am Orte der Dop-
} 
Es schien uns nicht ohne Interesse, noch mehr Bestätigungen jenes merkwürdigen theoretischen Satzes zu erhalten Es ist uns dies durch die Versuche, die nun beschrieben werden sollen, gelungen.

Wir machten einen dicken Platindraht in einer Flamme glühend und brachten ihn durch einen elektrischen Strom seinem Schmelzpunkte nahe. Der Draht gab ein glänzendes Spectrum ohne jede Spur von hellen oder dunkeln Linien. Wurde zwischen den Draht und den Spalt des Apparates eine Flamme von sehr wässerigem Alkohol gebracht, in dem Kochsalz aufgelöst war so zeigte sich die dunkle Linie $D$ in grosser Deutlichkeit.

In dem Spectrum eines Platindrahtes, der allein durch eine Flamme glühend gemacht ist, kann man die dunkle Linie $D$ hervorrufen, wenn man vor ihn ein Reagenzglas hält, auf dessen Boden man etwas Natriumamalgam gebracht hat, und dieses bis zum Kochen erhitzt. Dieser Versuch ist deshalb wichtig, weil er zeigt, dass weit unter der Glühhitze der Natriumdampf genau an derselben Stelle des Spectrums seine absorbirende Wirkung ausübt, wie bei den höchsten Temperaturen, welche wir hervorzubringen vermögen, und bei denjenigen, die in der Sonnenatmosphäre stattfinden.

Die helleren Linien der Spectren von $\mathrm{Ka}$, Sr, Ca, Ba umzukehren, ist uns gelungen bei Anwendung von Sonnenlicht und von Mischungen der chlorsauren Salze dieser Metalle mit Milchzucker. Vor dem Spalte des Apparates war eine kleine eiserne Rinne aufgestellt; in diese wurde die Mischung gebracht, volles Sonnenlicht längs der Rinne auf den Spalt geleitet und die Mischung durch einen glühenden Draht seitlich entzündet. Das Beobachtungsfernrohr war mit dem Schnittpunkt seiner schräg gestellten Fäden auf die helle Linie des Flammenspectrums, deren Umkehrbarkeit geprüft werden sollte, eingestellt; der Beobachter concentrirte seine Aufmerksamkeit darauf, zu beurtheilen, ob im Augenblicke der Verpuffung eine dunkle durch den Schnittpunkt des Fadenkreuzes gehende Linie sich zeigte. Auf diese Weise war es bei richtiger Mischung der abbrennenden Gemenge sehr leicht, die Umkehrbarkeit der Linien $B a \alpha$ und $B a \beta$ und der Linie $K a \beta$ zu constatiren. Die letzte von diesen fällt mit einer der deutlichsten, aber von FRAUNHOFER nicht bezeichneten, dunkeln Linie des Sonnenspectrums zusammen; diese Linie erscheint im Augenblicke der Verpuffung des Kalisalzes sehr viel deutlicher als sonst.

pellinie $D$ des Sonnenspectrums vorhanden sind, und dass der Bogen die dunkle Linie $D$ verstärkt oder erzeugt, wenn man durch ihn die Strahlen der Sonne oder einer der glühenden Kohlenspitzen gehen lässt und dann zu einem Spectrum auseinander legt. Die im Texte erwähnte Beobachtung giebt die Erklärung dieser interessanten, schon vor 11 Jahren von FOUCAULT bemerkten Erscheinung und zeigt, dass dieselbe nicht bedingt ist durch die Eigenschaften des in vieler Hinsicht noch so räthselhaften elektrischen Lichtes, sondern herrührt von einer Natriumverbindung, die in der Kohle enthalten war und durch den Strom in glühendes Gas verwandelt wurde. 
Um auf die beschriebene Weise die Umkehrung der hellen Linien des Strontiumspectrums zu sehen, muss der chlorsaure Strontian auf das Sorgfältigste getrocknet sein; eine Spur Feuchtigkeit bewirkt, dass bei der Verpuffung herumspritzende Salztheilchen die Flamme erfüllen, die Sonnenstrahlen dämpfen und das positive Strontiumspectrum zum Vorschein kommen lassen.

Wir haben uns in dieser Abhandlung darauf beschränkt, die Spectren der Metalle der Alkalien und alkalischen Erden und diese auch nur in so weit zu untersuchen, als es für die Analyse irdischer Stoffe nöthig ist. Wir behalten uns vor, diesen Untersuchungen die weitere Ausdehnung zu geben, die wünschenswerth ist in Beziehung auf die Analyse irdischer Körper und auf die Analyse der Atmosphären der Gestirne.

Heidelberg, im April 1860. 


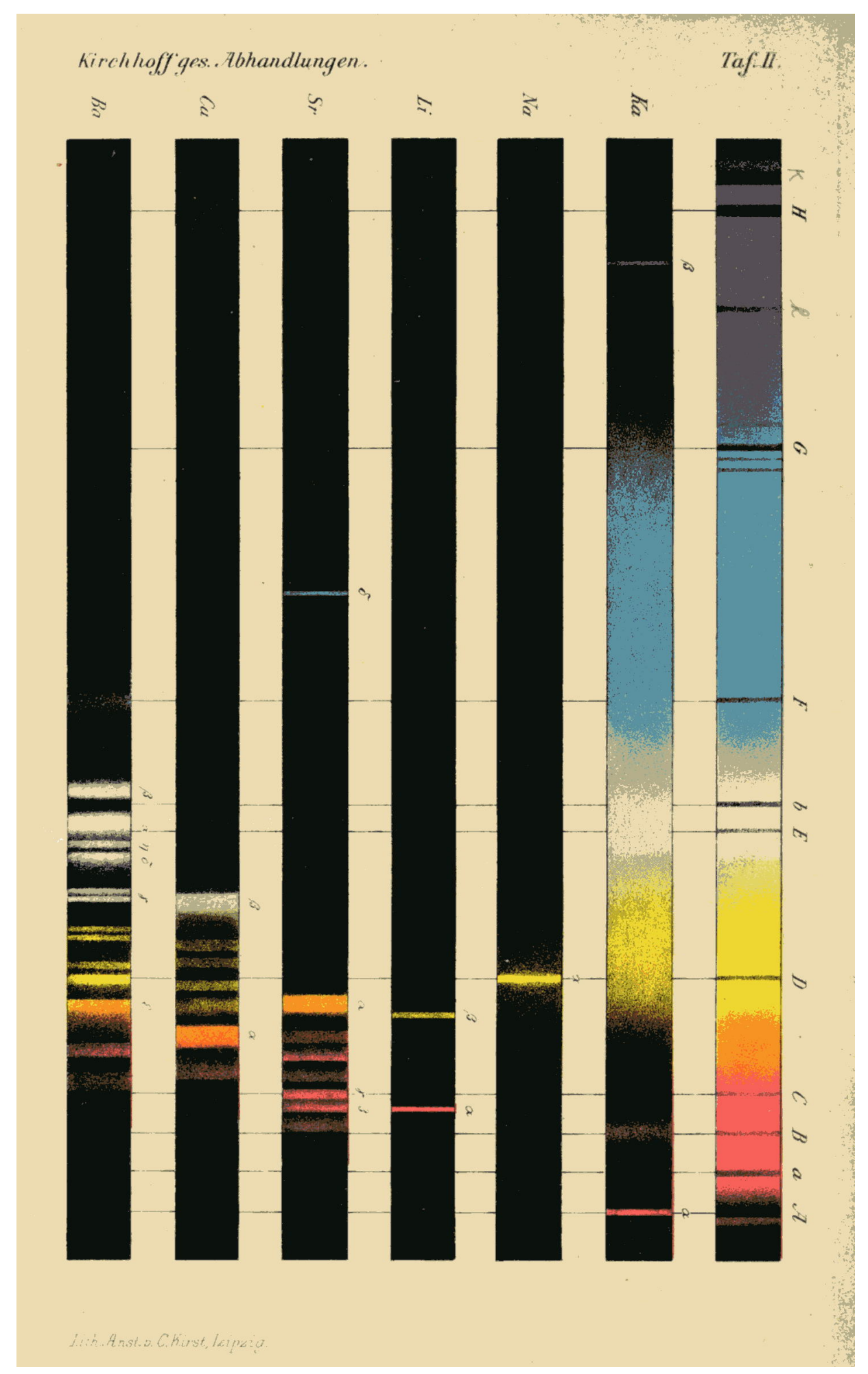

Abbildung 2: Spectren 